\title{
Metodología satelital litodinámica y escaneo de suelos para la verificación y/o identificación de nuevas fuentes de agua subterránea para uso de sistemas de agua potable rural
}

\author{
Methodology satellite lito dynamics and scanning of soils \\ for verification or identification of new sources of water underground \\ for the use of rural drinking water systems
}

Pedro Sariego $^{1 *} \quad$ Claudio Olguín $^{2} \quad$ Félix Pizarro $^{2} \quad$ Marcelo Muñoz $^{1}$

Recibido 11 de enero de 2016, aceptado 13 de junio de 2016

Received: January 11, 2016 Accepted: June 13, 2016

\begin{abstract}
RESUMEN
Los métodos convencionales de identificación de nuevas fuentes de agua muestran bajos indicadores de certeza, menores al 50\%, y en tiempos de escasez como los que vivimos, menores al $25 \%$, por consiguiente se hace necesario acudir a una nueva generación tecnológica de metodologías de búsqueda de nuevas fuentes de aguas subterráneas. El proyecto propone básicamente identificar un territorio de la región, desarrollar una búsqueda satelital que indique rastros de trazas de movimientos tectónicos milenarios que permitan identificar zonas ideales para escurrimientos o depósitos de agua subterránea, las que posteriormente deben ser escaneadas. Se propone un escaneo satelital a zonas específicas en la región, desarrollar el análisis litodinámico que tendrá como resultado la definición de "líneas de escurrimiento", sobre estas líneas se procede al escaneo de suelos, para cada línea se desarrollará un perfil, en algunos casos, de hasta 200 metros de profundidad, donde será posible identificar fuentes posibles de agua, cada uno de estos puntos será georreferenciado en la superficie. Un plan de perforaciones debidamente documentado mediante la información generada en el proyecto permitirá acceder a estas fuentes de agua detectadas. Un análisis paralelo a los elementos jurídicos del problema podrá permitir la inversión pública de los organismos correspondientes a nivel nacional, por consiguiente, el éxito del proyecto tendrá un enorme impacto social y económico, no solo en la región sino que en todo el país, se ha de tener presente que más del 15\% de la población nacional es abastecida de agua potable por sistemas de APRs.
\end{abstract}

Palabras clave: Escasez hídrica, agua potable rural, aguas subterráneas, nuevas tecnologías.

\section{ABSTRACT}

The conventional detection methods of new water sources are the named "hidrologycal studies", which display low indicators of certainty, below 50\%, and in times of shortage like the one we currently experiencing, are below 25\%. Therefore it's necessary to resort towards a new technological generation for new groundwater sources detection methodologies. This project proposes basically to recognize a territory inside the region, display a satelital search which signals for trails of traces of ancient tectonic movements that enable to identify ideal areas for runoffs or groundwater reservoirs, which should be scanned later. A satellite scanning intended to specific areas of the region, develop the lithodynamic analysis which will have as

1 Departamento de Ingeniería Mecánica. Universidad Técnica Federico Santa María. Casilla 110-V. Valparaíso, Chile. E-mail: pedro.sariego@usm.cl; marcelo.amunoz@gmail.com

2 Departamento de Ingenería Mecánica. Sede "José Miguel Carrera”. Universidad Técnica Federico Santa María. Casilla 110-V. Viña del Mar, Chile.E-mail: Claudio.olguin@usm.cl; felix.pizarro@usm.cl

* Autor de correspondencia 
result the "runoff lines" definition, on these lines we will proceed to scan the grounds, for each line we'll develop a profile, in some cases up to 200 meters deep, where it will be posible to identify potential water sources, each of these points will be geo-referenced on the surface. A drilling plan properly documented through the information generated across the venture, will enable to access these detected water sources. A parallel analysis to legal elements of the problem will may allow the public investment by corresponding bodies at a national level, accordingly, the project success will have a huge impact both social and economic, not only in the region but throughout the country, it must be remember that more $15 \%$ of the national population is supplied with drinking water through APRs systems.

Keywords: Water scarcity, rural water, underground water, new technologies.

\section{INTRODUCCIÓN}

La región de Valparaíso tiene declarada escasez hídrica en una seria de provincias especialmente en provincias de Petorca, Marga Marga y Quillota, extendiéndose a San Felipe, San Antonio, y comarcas de Valparaíso como Casablanca y Quinteros, solo en algunas comunas de la provincia de Los Andes aun no han decretado alguna vez escasez hídrica en los últimos cinco años. Lo anterior parece ir más allá pues el "Pronóstico de Disponibilidad de Agua para la Temporada de Riego 2014-2015" [1], presentado durante septiembre por la Dirección General de Aguas que establece a las regiones de Atacama, Coquimbo y Valparaíso como las más perjudicadas por el déficit de lluvias y nieve. Más aún, el tercer informe "Bajemos la Temperatura", publicado por el Banco Mundial [6], advierte que condiciones como las olas de calor y otros fenómenos extremos que se producen cada 100 años o más, y que en la actualidad se consideran como inusuales, se convertirán en la "nueva realidad climática". Por consiguiente, más que a una sequía puntual (como la de los años 1994-1995, 2007-2008), es un estado de emergencia específico, los expertos tienden a pensar que la región enfrenta un proceso de cambio climático que está afectando en lo inmediato con fuerza todos los sistemas de agua potable rural y el productivo agrícola de la región, declarándose fuertes conflictos entre privados, comunidades de agua potable rural, y exigencias al aparato del Estado y en especial a las autoridades de la región, como asimismo a las organizaciones y unidades vinculadas a la inversión pública y/o fiscalización con el tema del agua. Al respecto, la Tabla 1 indica los impactos producidos por la sequía extendida en el tiempo en un determinado territorio, que ha sido complementado por el equipo de presentación de esta propuesta, la cual sintetiza a lo que se expone una región sino tiene capacidad de solucionar los problemas por falta de agua, no solo en el sector rural sino que también urbano.

\section{Área objetivo: Comunidades de agua potable rural} En este sentido, en líneas generales la cobertura de agua potable rural ha sido un desafío de larga data

Tabla 1. Identificación de impactos producidos por las sequías [2].

\begin{tabular}{|l|l|l|}
\hline \multicolumn{1}{|c|}{ Económicos } & \multicolumn{1}{c|}{ Sociales } & \multicolumn{1}{c|}{ Ambientales } \\
\hline $\begin{array}{l}\text { Impactos en la productividad agrícola } \\
\text { y forestal. }\end{array}$ & $\begin{array}{l}\text { Impactos en la higiene y salud personal } \\
\text { y pública. }\end{array}$ & Erosión de suelos. \\
\hline Impactos en la productividad ganadera. & Incremento en las tasas de desempleo. & Incendios forestales. \\
\hline $\begin{array}{l}\text { Impactos en la producción hidráulica } \\
\text { (energía). }\end{array}$ & $\begin{array}{l}\text { Deterioro o pérdida de espacios para } \\
\text { recreación. }\end{array}$ & $\begin{array}{l}\text { Degradación de la calidad del agua debido } \\
\text { a la concentración de contaminantes. }\end{array}$ \\
\hline $\begin{array}{l}\text { Impactos en los costos de tratamientos } \\
\text { y provisión de agua potable. }\end{array}$ & $\begin{array}{l}\text { Incremento de los índices de mortalidad } \\
\text { y morbilidad. }\end{array}$ & Deterioro de la calidad visual del paisaje. \\
\hline $\begin{array}{l}\text { Impactos en las actividades industriales } \\
\text { y comercial. }\end{array}$ & Violencia desatada. "guerra del agua". & Efecto sobre la flora y fauna autóctona. \\
\hline Impacto sobre la actividad educativa. & & $\begin{array}{l}\text { Proceso de desertificación. } \\
\text { mínimos ecológicos. }\end{array}$ \\
\hline Impacto sobre el turismo. & & \\
\hline
\end{tabular}


en Chile. Como señala la historia del Programa de Agua Potable Rural (APR), "al comienzo de la década de 1960, tan solo el $6 \%$ de los habitantes de las localidades rurales de Chile contaba con agua potable. Hoy esa cobertura de más de $99 \%$ en las localidades concentradas, que corresponden a aquellas con más de 150 habitantes y una densidad mayor a 15 viviendas por kilómetro de red de agua potable.

Parte de los sistemas habilitados son apoyados y supervisados por la Dirección de Obras Hidráulicas del Ministerio de Obras Públicas, y menor proporción por las municipalidades. Al respecto hacia el 2013 que la población nacional abastecida por el Programa APR adscrito a la DOH era de aproximadamente 1.580 .000 personas distribuidas en 1.631 comités y cooperativas en todo el territorio nacional. Se estima que 1.150 .000 se encuentran en zonas rurales.

Cuando falla el abastecimiento de agua de pozo, llegan los camiones aljibes con todo su triste significado. Las consecuencias de no poder abastecer de agua al ganado y que decir de la agricultura familiar, asociados a estas comunidades son una

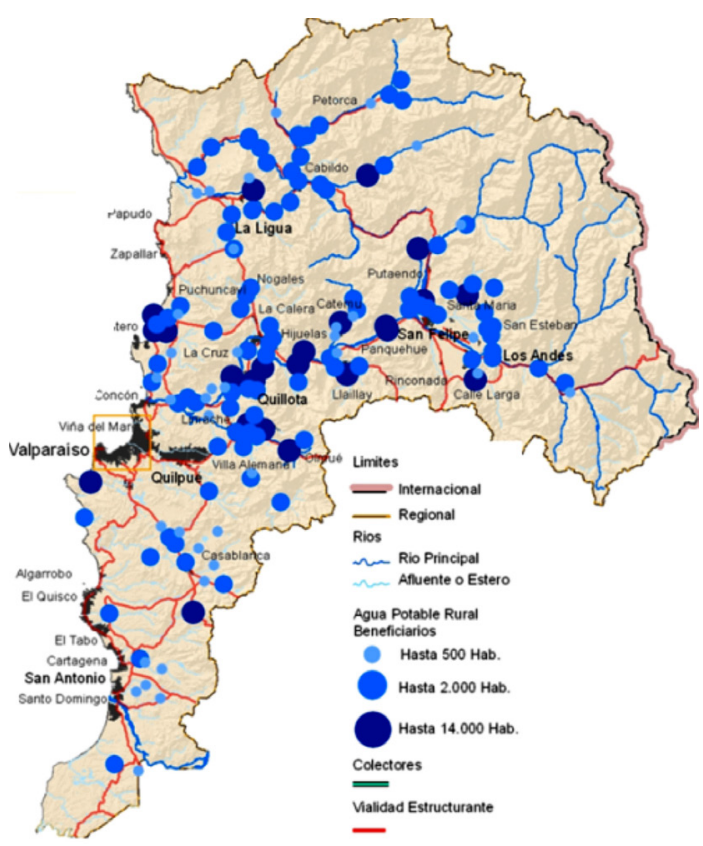

Fuente: Elaboración Dirplan Regional, información $\mathrm{DOH}$

Figura 1. Ubicación territorial de las comunidades beneficiarias de APRs en la región de Valparaíso, identificadas por tamaño relativo [5]. profunda crisis socioeconómica que las lleva a la crisis y en muchos casos a la emigración.

Lo cierto es que al agotamiento de las napas conocidas, que dicho sea de paso requerirán años y años de lluvias en excesos para recuperar sus niveles normales, supone la búsqueda de otras que les reemplacen pues la profundización de pozos está alcanzando un punto en que ya no es solución.

\section{Método tradicional}

Para la identificación de nuevas fuentes de aguas subterráneas, la técnica aceptada por el sistema nacional de inversiones es el estudio hidrogeológico a los acuíferos, que básicamente están constituidos por formaciones geológicas compuestas por materiales permeables y porosos que reciben el agua, la almacenan y permiten el tránsito de la misma. Los acuíferos pueden ser de tipo fisurado (fracturas en rocas cristalinas como granitos) o granulares (sedimentos de tipo arenas, etcétera). A su vez estos últimos pueden ser: libres (a presión atmosférica) o confinados (a presión mayor que la atmosférica por lo que pueden ser surgentes). El agua subterránea se explota mediante perforaciones en el terreno. Las mismas se ubican como resultado de un estudio hidrogeológico previo, y también en función de ese estudio y de las muestras de subsuelo extraídas durante la perforación del terreno, es que se diseña la construcción de la misma. La cuestión es que es el nivel de certeza del estudio hidrogeológico, especialmente en la condición de sequía en la región, el que representa un bajo nivel de asertividad, ya en el 2013, la DOH aseveraba que esa certeza era menor que el $25 \%$ en la provincia de Petorca. Por consiguiente, se requiere ser más asertivo en la identificación de esas fuentes de agua.

\section{La nueva generación de métodos}

Dentro de estos métodos es posible hacer una diferenciación entre aquellos métodos de fuente natural y los de fuente artificial; los primeros miden las variaciones energéticas naturales provenientes directamente de fuentes geológicas, mientras que los de fuente artificial como su nombre lo indica, miden la respuesta a la excitación provocada por una fuente externa. Ver Tabla 2.

Para clasificar los métodos geofísicos de prospección hidrogeológica se utilizará como criterio el tipo de fuente energética del que provienen las excitaciones que miden. Si bien existen otros criterios que pudieran 
Tabla 2. Clasificación de métodos [4].

\begin{tabular}{|c|l|}
\hline \multirow{4}{*}{$\begin{array}{c}\text { Fuente natural } \\
\text { (pasiva) }\end{array}$} & Gravimetría: Medición de la interacción del campo gravitatorio de la tierra. \\
\cline { 2 - 2 } & Radiometría: Emisión electromagnética de isótopos radioactivos naturales. \\
\cline { 2 - 2 } & Magnetometría: Medición del campo magnético de la tierra. \\
\cline { 2 - 2 } & Electromagnético: Acciones naturales como relámpagos y actividad solar. \\
\hline \multirow{2}{*}{$\begin{array}{c}\text { Fuente artificial } \\
\text { (activa) }\end{array}$} & Eléctricos: Excitación por medio de corriente continua. \\
\cline { 2 - 2 } & Electromagnéticos: Excitación por medio de generadores electromagnéticos. \\
\cline { 2 - 2 } & Sísmicos: Generación artificial de energía sísmica. \\
\hline
\end{tabular}

utilizarse como, por ejemplo, el medio físico de transmisión (eléctrico, magnético, etcétera), el uso de fuentes externas genera un considerable aumento de la logística en terreno y por cierto de los costos. Si bien existen variaciones y combinaciones de estos métodos, como por ejemplo técnicas geofísicas de Teledetección y Aerotransportadas, el principio es el mismo y puede clasificarse dentro de la lista de más arriba. Los avances más significativos de las últimas décadas son principalmente de tipo instrumental y técnicas de tratamiento de datos. Es así como algunas de las técnicas descritas en el capítulo anterior, han sido mejoradas con herramientas como los elementos finitos y software de procesamiento de datos de gran capacidad.

Dentro de este último grupo se encuentran los métodos geofísicos de fuente artificial de mayor complejidad pero que presentan la mayor versatilidad y ventajas en comparación a sus predecesores, los de mayor impacto en los últimos años dos indican a continuación.

\section{Propuesta "Satelital, litodinámico y escaneo de suelos"}

De acuerdo a la norma NCH ISO 9000:2008, se define VALIDACIÓN como confirmación, por medio de la provisión de evidencia objetiva de que los requisitos para intenciones o aplicaciones de uso específico se han cumplido. Las condiciones para desarrollar la validación pueden ser reales o simuladas.

El Departamento de Ingeniería Mecánica de la Universidad Técnica Federico Santa María, liderará el proceso de validación, desarrollará el marco conceptual de la metodología, dará sustento estadístico al proyecto, establecerá las medidas pertinentes de coordinación con las entidades de apoyo técnico y jurídico del proyecto, la red de apoyo con las instituciones públicas y privadas, entre otros, finalmente desarrollará un intenso despliegue comunicacional de esta metodología por la importancia que tiene para todo el país. La fase de validación propiamente tal incluirá la definición de costos y la identificación de nuevas oportunidades de innovación en el tema del agua en la región. Las pruebas satelitales litográficas y escáner de suelo se llevarán a cabo a través de empresas especializadas, que poseen el conocimiento del tema a la fecha en Chile y cuyas principales prospecciones han estado asociadas a la fecha en el mundo minero, las pruebas de perforación serán desarrolladas por medio de una empresa contratista dedicada a este tipo de labores en la región y finalmente el análisis jurídico será encargado a un abogado especialista con conocimiento profundo de la cuestión hídrica de la región.

La innovación consiste en validarla como método para la búsqueda de nuevas fuentes de agua ante el sistema de inversión pública nacional, con niveles de certeza superiores a los que presentan los métodos convencionales hidrogeológicos. La descripción del trabajo a realizar en estas zonas se desglosa en: un estudio satelital litodinámico, que se propone en esta propuesta se realice en cinco áreas del territorio de la región, de aproximadamente $9 \mathrm{~km}^{2}$ a una escala de 1:50.000. En esta etapa se realiza un mapa generalizado de los grandes sistemas de flujo y la secuencia de grandes estructuras geológicas y tectónicas con el objeto de determinar la posición de los lugares de mayores perspectivas en relación con los sistemas de flujo y otras estructuras, identificando la existencia de estructuras tectónicas circulares y lineales de gran escala.

El objetivo es para crear el modelo geológico y geométrico, basado en el concepto de paleocanales, derivado mediante el método de paleorreconstrucción. El método en específico se basa en la creación de mapas que identifican las antiguas estructuras litodinámicas, paleoflujos, estructuras de tipo circular, y otras características, que ayudan a 
evaluar la migración de fluidos y la acumulación. La paleorreconstrucción usa un método físico matemático que fue desarrollado hace más de doscientos años por famosos matemáticos como Leibnitz, Gauss y Newton. A pesar de que este método es una aplicación relativamente nueva en la exploración de recursos minerales, el método de paleorreconstrucción tiene más de veinte años de aplicaciones exitosas y ha demostrado su utilidad en la predicción de la presencia de hidrocarburos minerales y aguas subterráneas en el extranjero. Debido a que el método genera un mapa que muestra la apariencia de "cómo fluyen los fluidos", el mismo describe los mapas resultantes de la superficie como un mapa de "plasticidad de relieve". Las tareas anteriores se resuelven por medio de aplicación de software informático especializado dando como resultado la construcción de los mapas de plasticidad de relieve que reflejan ubicación de las áreas potenciales de aguas subterráneas o estructuras, en este caso serán de interés la definición de las denominadas "Líneas de Agua" a escala 1:10.000, que permite la búsqueda con escáner de suelo de los perfiles en las líneas de interés determinando las estructuras favorables para la presencia de aguas subterráneas.

En conjunto con la información obtenida de los estudios litodinámicos, geofísicos e hídricos que existan en la zona de estudio, se han de identificar los puntos de realización de pozos de sondaje experimentación para dar certeza de agua. Esto se hace caminando por las líneas de agua previamente, lanzando pulsos electromagnéticos de baja frecuencia a alta potencia para trabajar en ambientes de alta conductividad como el barro o la arcilla húmeda, grabando sus reflexiones sobre las interfaces entre las capas u objetos, generando como producto el perfil deseado hasta 200 metros de profundidad y especificando los puntos de interés geo referenciados en su nivel.

\section{Hitos del proyecto en desarrollo}

En resumen, el proyecto ha de desarrollar los siguientes hitos. La constatación de los mismos constituyen los medios de verificación en terreno. La primera etapa, que corresponde a los estudios litodinámicos. Se ha de entregar un informe que indicará zonas de interés dentro del área, y posteriormente se identificarán las líneas de agua a recorrer. La segunda etapa, que corresponde a los estudios geofísicos en terreno (escáner de suelos) recorriendo las líneas de agua, el resultado será un perfil geológico de la línea, con una profundidad de 200 metros. Una tercera fase es el análisis de estos perfiles que establece los puntos de interés georreferenciados. En cada una de las áreas investigadas se especificará el tipo de suelos, definiendo un plan de factibilidad de sondajes, se ha de tener presente que se tiene una gran experiencia en Chile en suelos sedimentados pero no en suelos de rocas fracturada. Por consiguiente, para cada área explorada se tendrá un mapa de suelos escaneado, con el que la autoridad correspondiente podrá desarrollar un plan de prospecciones en la región. La última etapa de la fase exploración del método, que consiste en la interpretación de los datos obtenidos en las etapas anteriores y confección de un informe final. Por último, a fin de poder invertir sin las menores trabas burocráticas de parte del Estado, tanto para el caso de los APR, como para caso del consumo del agua para los animales como para el riego, es que se hace necesario hacer un análisis amplio de tipo jurídico que abarque desde temas generales a normativas reglamentarias. En este contexto se ubican normativas como el DFL 850 del Ministerio de Obras Públicas, que tendrá bastante injerencia en el desarrollo de las obras efectuadas por ese ministerio.

\section{CONCLUSIONES}

El proyecto se encuentra en pleno desarrollo en áreas seleccionadas en seis comunas de la región, a saber: Petorca y La Ligua en la Provincia de Petorca; Nogales e Hijuelas en la Provincia de Quillota; Santa María en la Provincia de San Felipe y San Esteban en la Provincia de Los Andes. La condición de selección de los mismos fue que se tratara de terrenos fiscales, ojalá aledaños a Sistemas de APRs con problemas. El conocimiento del territorio por parte del equipo de trabajo ha sido determinante del éxito de elección de las áreas de interés para el proyecto. Los análisis prospectivos desarrollados a la fecha indican que las hipótesis indicadas son ciertas, con lo cual se esperan auspiciosos resultados que muestran la necesidad de entrar en una fase de contención de las expectativas de las comunidades cercanas a la fase de exploración. Sin embargo, el análisis jurídico del tema ha encontrado más dificultades de las esperadas debido a que la institucionalidad del agua se encuentra repartida en diversas reparticiones del Estado. 
Este es un trabajo de investigación aplicado que da cuenta de la interacción de la ingeniería mecánica con la comunidad y el aparato público en un tema estratégico para el desarrollo de un territorio. Si no hay agua no hay actividad productiva, más aún no hay asentamiento humano, por consiguiente, lo que está en juego va más allá de una aplicación específica del conocimiento de un área de la ingeniería mecánica. Participar y aportar desde el conocimiento y experiencia que un profesional de la ingeniería ha desarrollado en el tiempo a iniciativa como esta es ante todo un deber ciudadano, hacerlo con un equipo de trabajo más allá de la especialidad es un acierto y un signo de los tiempos, llevarlo a cabo para comunidades en riesgo social, todo un privilegio.

\section{AGRADECIMIENTOS}

Esta iniciativa ha sido financiada con aportes del Fondo de Innovación para la Competitividad del Gobierno Regional de Valparaíso año 2015.

\section{REFERENCIAS}

[1] Dirección General de Agua (DGA). Ministerio de Obras Públicas (MOP). "Pronóstico de Disponibilidad de Agua para la Temporada de Riego 2014-2015”. DGA/MOP. Santiago, Chile. 2013.

[2] G. Donoso. "Las Sequías en Chile: Causas, Consecuencias y Mitigación”. Capítulo "Impacto econométrico de las sequías sobre la agricultura en Chile", pp. 55. Editorial PUC. Santiago, Chile. 1999.

[3] P. Sariego. Presentación Proyecto FIC 2015 Gobierno Regional de Valparaíso, "Validación de metodología satelital litodinámica y escaneo de suelos para la verificación y/o identificación de nuevas fuentes de aguas subterráneas para uso de Sistemas de Agua Potable Rural". UTFSM. Valparaíso, Chile. 2015.

[4] M. Muñoz. "Análisis de métodos de prospección de agua subterráneo para consumo humano en zonas rurales sometidas a escasez hídrico". Trabajo de título Ingeniería Civil Mecánica. UTFSM. Valparaíso, Chile. 2015.

[5] Ministerio de Obras Públicas Región de Valparaíso. "Plan Regional de Infraestructura y Gestión del Recurso Hídrico al 2021". Resumen Ejecutivo. MOP. Valparaíso, Chile. 2012.

[6] Grupo Banco Mundial. URL: http://www. bancomundial.org/es/topic/climatechange/ publication/turn-down-the-heat 